\title{
Family-Based Study of AVPRIB Association and Interaction with Stressful Life Events on Depression and Anxiety in Suicide Attempts
}

\author{
Yair J Ben-Efraim', Danuta Wasserman ', Jerzy Wasserman' and Marcus Sokolowski*,' \\ 'The National Centre for Suicide Research and Prevention of Mental III-Health (NASP), Karolinska Institute (KI), Stockholm, Sweden
}

\begin{abstract}
The cortisol response to psychosocial stress may become dysregulated in stress-related disorders. It is potentiated by pituitary secretion of adrenocorticotropic hormone (ACTH), which is, in part, regulated by arginine vasopressin receptor-IB (AVPRIB). AVPRIB variants were previously reported to associate with mood and anxiety disorders. This study aims, for the first time, to investigate association of AVPR IB genetic variants with mood and anxiety outcomes in suicidal behavior. Using a family-based study design of 660 complete nuclear family trios with offspring who have made a suicide attempt (SA), we tested the direct association and linkage of AVPRIB single nucleotide polymorphisms (SNPs) with SA, as well as with depression and anxiety in SA. Main findings were the association and linkage of AVPRIB exon I SNP rs33990840 and a major 6-SNP haplotype representative of all common AVPR IB-SNPs, on the outcome of high Beck Depression Inventory scores in SA. By contrast, genetic associations with lifetime diagnoses of depression and anxiety in SA or gene-environment interactions between AVPRIB variants and stressful life events (SLEs) were not significant. An exploratory screen of interactions between AVPRIB and CRHRI (corticotropin-releasing hormone receptor-I), the principal pituitary regulator of $A C T H$ secretion, showed no support for gene-gene interactions on the studied outcomes. The results suggest that AVPRIB genetic variation, eg, non-synonymous SNP rs33990840 mediating putative consequences on ligand binding, has a role in SA etiology characterized by elevated depression symptoms, without involving AVPR IB-moderation of SLEs.

Neuropsychopharmacology (2013) 38, I504-15II; doi:I0.1038/npp.20।3.49; published online 20 March 2013
\end{abstract}

Keywords: arginine vasopressin; CRHRI; depression; G×E; HPA axis; stress; suicidality

\section{INTRODUCTION}

A dysregulated cortisol response to psychosocial stress is often observed in suicidal behavior, mood, and anxiety disorders and may be assessed by dexamethasone (DEX) non-suppression, one of the best known biological predictors of completed suicide in depression (Mann et al, 2006). Although corticotropin-releasing hormone (CRH) signaling via CRH receptor-1 (CRHR1) is the principal activation pathway of the stress-reactive hypothalamicpituitary-adrenal (HPA) axis, arginine vasopressin (AVP) signaling via AVP receptor-1B (AVPR1B) may act synergistically in response to certain stressors (Scott and Dinan 1998). Furthermore, CRH challenge combined with DEX (CRH/DEX), which is putatively more sensitive in detecting hypothalamic AVP dysregulation than DEX, has also been used in assessing stress-related disorders, eg, depression (Bardeleben and Holsboer 1989; Purba et al, 1996). Increased AVP signaling in HPA axis stress reactivity has

*Correspondence: Dr M Sokolowski, National Centre for Suicide Research and Prevention of Mental III-Health (NASP), Karolinska Institute (KI), Stockholm S-I7I 77, Sweden. Tel: + 46852486938 , Fax: + 4683 06439, E-mail: marcus.sokolowski@ki.se

Received 17 August 2012; revised 8 February 2013; accepted II February 2013; accepted article preview online 19 February 2013 additionally been shown to occur following exposure to chronic psychosocial stressors, which are commonly thought to underlie chronic stress-related disorders, eg, depression (Scott and Dinan 1998; Bao and Swaab 2010).

Single nucleotide polymorphisms (SNPs) and haplotypes in AVPR $1 B$ were previously observed to associate with stressrelated mood and anxiety disorders (van West et al, 2004; Dempster et al, 2007; Keck et al, 2008; van West et al, 2009; Leszczynska-Rodziewicz et al, 2012). AVPR1B knockout in animal models suggest that the gene is involved in response to multiple stressors, in addition to chronic stressors (Roper et al, 2011). This genetic evidence is supported by a reduction in stress-related outcomes in response to $A V P R 1 B$ pharmacological blockade in animal models, and antagonists are currently in development for stress-related disorders (Griebel et al, 2005; Roper et al, 2011).

However, the precise role of $A V P R 1 B$ genetic variation in stress reactivity and treatment outcomes in stress-related disorders is still unclear. In two recent studies, $A V P R 1 B$ SNPs did not moderate a reduction in depressive symptoms in response to citalopram (Binder et al, 2010) or cortisol response to a socio-evaluative stressor (van West et al, 2010). Evidence for variation in other HPA axis candidate genes,eg, CRHR1, has been more consistent across studies of cortisol response, suicidality, mood, and anxiety disorders (Binder 
and Nemeroff 2010). To date, there has been no study on gene-environment interaction $(\mathrm{G} \times \mathrm{E})$ between $A V P R 1 B$ variants and stressful life events (SLEs) on any outcome.

In line with previous evidence of AVP/AVPR1B stressrelated signaling in animals and humans (Scott and Dinan 1998; Bao and Swaab 2010), G $\times$ Es may be hypothesized between $A V P R 1 B$ variants and SLEs represented by chronic/ repeated and interpersonal, network-related psychosocial stressors, on the outcome of suicide attempt (SA), as well as depression and anxiety in SA. Although $A V P R 1 B$ and CRHR1 are not expressed exclusively in HPA axis, a potential role for $A V P R 1 B$ variation in HPA axis activation might also be observed by interaction with $C R H R 1$ variants that associated with cortisol response. Variants of $A V P R 1 B$ and CRHR1 were previously shown to have combined genetic effects in association with panic disorder, suggesting the potential for observing gene-gene interaction effects $(\mathrm{G} \times \mathrm{Gs})$ in adequately powered studies (Keck et al, 2008). Moreover, $A V P R 1 B$ and CRHR1 are capable of physically interacting (Young et al, 2007), and heterodimerization affects their pharmacological properties in vitro (Murat et al, 2012). However, heterodimerization in vivo or $\mathrm{G} \times \mathrm{Gs}$ on stress-related outcomes have not been shown.

To complement and extend previously published $A V P R 1 B$ associations on mood and anxiety disorders, we tested here two succeeding hypotheses in a family-based association study. The primary hypothesis of our study was that (i) $A V P R 1 B$ is a candidate gene of interest in overall SA etiology, or in the etiology of a subset of SA outcomes characterized by depression, anxiety, and/or sex, whereby SNPs were expected to directly associate with those outcomes. We then continued with testing the second hypothesis of (ii) interactions between $A V P R 1 B$ genetic variants and SLEs on those SA outcomes, by using $\mathrm{G} \times \mathrm{E}$ analyses. Finally, we also wished to characterize the influence of other non-hypothesized factors on any associations identified, as well as to conduct an exploratory screen for putative $\mathrm{G} \times \mathrm{Gs}$ between $A V P R 1 B$ and CRHR1 SNPs on the studied SA outcomes.

\section{SUBJECTS AND METHODS}

\section{Research Subjects}

Research subjects were collected in Ukraine in 2001-2006, numbering 660 complete and extensively quality-controlled nuclear family trios with SA offspring and 519 healthy volunteers (HVs), as described previously (Wasserman et al, 2005; Ben-Efraim et al, 2011). Of the 267 trios previously analyzed in our initial publication (Wasserman et al, 2005), 199 trios were also used in the present analysis of 660 trios (68 trios omitted due to DNA-concentration incompatibilities with the currently used genotyping platform). The collection of research subjects followed the code of ethics of the World Medical Association (Declaration of Helsinki), and a written consent was obtained. The study was approved by the Research Ethics Committee at the Karolinska Institute (Dnr 97-188) and by the Ministry of Health in Ukraine. Selection criteria, sample demographics, International Classification of Diseases 10th edition (ICD-10) psychiatric diagnoses, and DNA preparation and genotyping have been described previously for trios (Wasserman et al, 2008) and HVs
(Ben-Efraim et al, 2011). Briefly, SA offspring were Ukrainian citizens with $94.1 \%$ having $\geqslant 3$ grandparents of Ukrainian/ Russian ethnicity, $51.1 \%$ were male and $48.9 \%$ were female, with mean ages of $24.6(\mathrm{SD} \pm 7.3)$ and $23.8(\mathrm{SD} \pm 7.1$ ) years, respectively. HVs were unrelated to trios with $93.8 \%$ having $\geqslant 3$ grandparents of Ukrainian/Russian ethnicity, $45.9 \%$ were male and $54.1 \%$ were female, with mean ages of 35.7 $(\mathrm{SD} \pm 16.1)$ and $34.6(\mathrm{SD} \pm 14.8)$ years, respectively. Anxiety, depression, and depressive symptoms are frequently observed to associate with a dysregulated HPA axis as well as suicidal behavior and may be intermediate outcomes linking other diagnoses, eg, alcohol use disorders and psychoses, respectively, with suicidal behavior (Lönnqvist 2009). Therefore, we did not exclude subjects with any specific psychiatric diagnosis before analysis.A more comprehensive description is also provided (Supplement).

\section{Polymorphisms}

We selected $13 A V P R 1 B$ SNPs for genotyping (SNP\&SEQ Technology Platform, Uppsala, Sweden), according to SNPs previously identified in association studies on panic disorder and recurrent major depression (van West et al, 2004; Keck et al, 2008) or in dbSNP (http://www.ncbi. nlm.nih.gov/snp) in putatively functional positions such as promoter, untranslated regions, or exons (Figure 1a). One genotyped SNP was monomorphic (rs35775245, all CC homozygotes) and therefore removed from further analysis. The remaining 12 genotyped SNPs (Table 1) were in Hardy-Weinberg Equilibrium $(P>0.05)$, had high call rates $(\geqslant 99.8 \%)$, absence of mendelian inheritance errors, and $100 \%$ intra-assay reproducibility.We then proceeded to cover as much of the coding and flanking regions as possible by imputation of untyped 'common' SNPs $(\mathrm{MAF} \geqslant 0.05)$ with a European ancestry reference (EUR) panel (The 1000 Genomes Project Consortium 2010) using software BEAGLE v3.3 (Browning and Browning 2009), as described previously (Ben-Efraim et al, 2012) and modified here for the novel reference panel (Supplement). After postimputation processing and linkage disequilibrium (LD)pruning as previously described (Ben-Efraim et al, 2012), four common genotyped (non-imputed) SNPs and one common imputed SNP remained for analysis (Figure 1 and Table 1). The four genotyped (non-imputed) SNPs and one imputed SNP (BEAGLE allelic $r^{2}=1$ ) tagged by high LD $\left(r^{2}\right.$ threshold $\left.=0.80\right)$ all the 38 other (either genotyped or imputable) common SNPs (with MAF $\geqslant 0.05$ ) present in the $A V P R 1 B$ coding region, flanking $5 \mathrm{~kb}$ upstream and $0 \mathrm{~kb}$ downstream of the untranslated regions (Table 1). Any associations to be discovered with these five selected SNPs could thus be confirmed retrospectively to exist also for the other corresponding SNPs being tagged (Table 1). In addition, one genotyped SNP with low-frequency $(\mathrm{MAF}=0.019) \quad$ was included in analysis (rs36046834; Figure 1). For $\mathrm{G} \times \mathrm{G}$ investigation of $A V P R 1 B \times C R H R 1$, five $C R H R 1$ variants were selected out of a larger genotyped panel according to previous associations with cortisol level changes, depression, anxiety, or suicidality (Supplement).

\section{SLEs}

Environmental exposures were assessed as described previously (Ben-Efraim et al, 2011). SLE types were either 

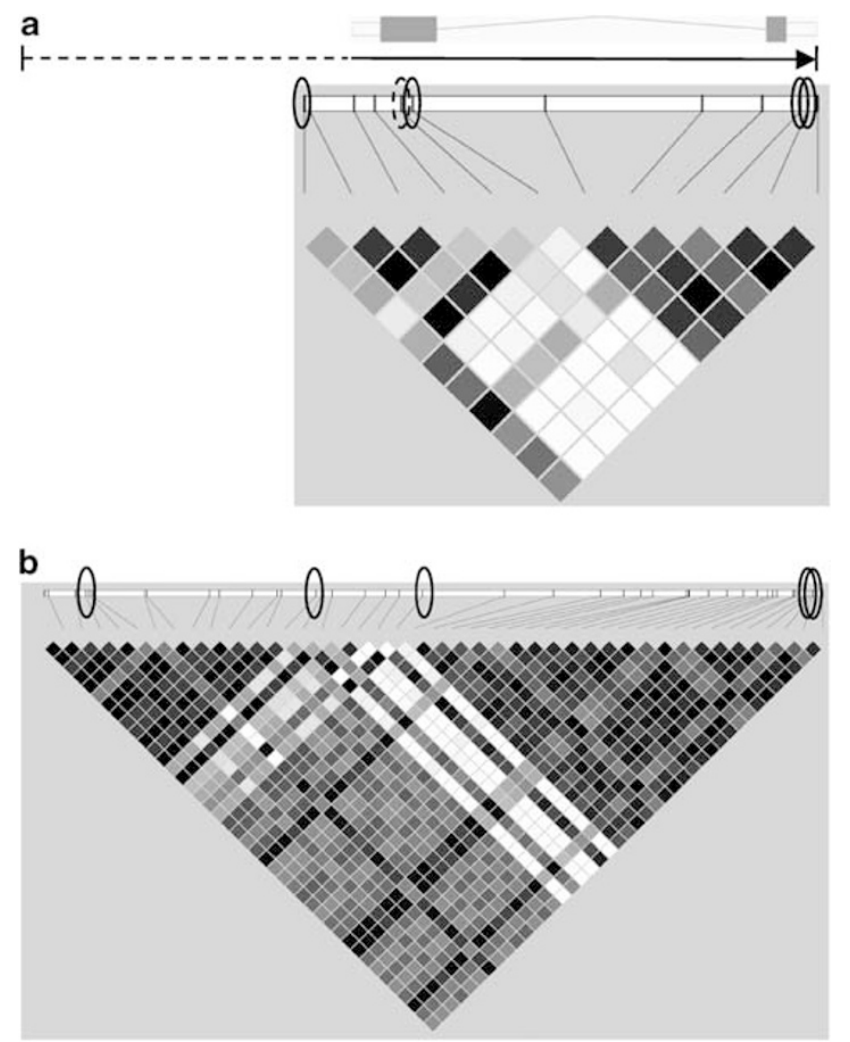

Figure I Schematic diagram of the gene and linkage disequilibrium map. Above: Schematic of AVPRIB on the forward strand of chromosome I. Ensembl (release 66) protein coding region showing untranslated regions (clear boxes), two exons (filled boxes) separated by one intron (line), with a solid arrow marking the coding region and direction of transcription ( $5^{\prime}$ to $\left.3^{\prime}\right)$. The coding region is $7.66 \mathrm{~kb}$ long, and the dashed line marks this study's coverage of common single nucleotide polymorphisms (SNPs) in the $5^{\prime}$ flanking region $(5.03 \mathrm{~kb})$. Below: Linkage disequilibrium (LD; $\left.r^{2}\right)$ map showing genotyped SNPs relative to their position on the gene. Solid circles mark the position of tag SNPs used in the study, and the dashed circle marks the position of a low-frequency exonic SNP. (a)Twelve genotyped SNPs. Four-tag SNPs cover $92 \%$ of common variation within the coding region, and a distance of $12.70 \mathrm{~kb}$, including the $5^{\prime}$ flanking region (EUR panel). Untagged common SNPs were rs35369693 and rs35810727. (b) Forty-three common SNPs post-imputation. Five-tag SNPs cover $100 \%$ of common SNP variation in the coding region,and the same total distance. Four-tag SNPs are the same as in panel (a), and an additional nongenotyped, imputed tag SNP is marked by the left circle.

studied alone or aggregated according to exploratory assessment of exposure to chronic/repeated and interpersonal, network-related psychosocial stressors (Supplement). Overall, SLEs analyzed as dichotomous variables were: (1) childhood/adolescence physical assault, (2) childhood/ adolescence sexual assault, (3) lifetime SLEs checklist (median-split), (4) lifetime SLEs checklist network subscale (median-split), (5) repeated assault (physical or sexual assault in both childhood/adolescence and adulthood), and (6) childhood/adolescence assault and median-or-greater ('high') lifetime SLEs.

\section{Outcome Measures}

Trio subjects were interviewed in their home environment within 4 weeks (median 11 days) after the ascertained index SA. All index SA had a score of $\geqslant 2$ on the Medical Damage
Table I The Five SNPs Selected for Analysis Captured I00\% of the Common SNP Variation in AVPRIB

\begin{tabular}{|c|c|c|c|c|c|}
\hline \multirow{2}{*}{$\begin{array}{l}\text { AVPRIB } \\
\text { SNP }\end{array}$} & \multirow{2}{*}{$\begin{array}{l}\text { Major and } \\
\text { minor } \\
\text { alleles }\end{array}$} & \multicolumn{2}{|c|}{ MAF } & \multirow{2}{*}{$\begin{array}{l}\text { Base pair } \\
\text { position }^{\mathrm{a}}\end{array}$} & \multirow{2}{*}{$\begin{array}{c}\text { Position } \\
\text { relative to } \\
\text { coding region }\end{array}$} \\
\hline & & gt & pi & & \\
\hline rs35630000 & $\mathrm{C} / \mathrm{T}$ & NA & 0.144 & 206219605 & $5^{\prime},>2 \mathrm{~kb}$ \\
\hline rs339||258 & $\mathrm{T} / \mathrm{C}$ & 0.177 & 0.177 & 206223297 & $5^{\prime}, \leqslant 2 \mathrm{~kb}$ \\
\hline rs33990840 & $\mathrm{G} / \mathrm{C}$ & 0.073 & 0.074 & 206225011 & Exon I (non-syn) \\
\hline rs28529|27 & $\mathrm{G} / \mathrm{A}$ & 0.106 & 0.106 & 206231152 & $3^{\prime}-$ UTR \\
\hline rs33933482 & G/A & 0.124 & 0.125 & 206231264 & $3^{\prime}-U T R$ \\
\hline
\end{tabular}

Abbreviations: gt, genotyping (non-imputed) minor allele frequency (MAF); NA, not available due to single nucleotide polymorphism (SNP) being imputed (and not genotyped); non-syn, non-synonymous coding variant; pi, post-imputation MAF; UTR, untranslated region.

Notes: Additionally, one genotyped and rare (MAF $=0.017)$ SNP rs36046834 was analyzed in the study (major and minor alleles G/C, base pair position 206224830), as well as haplotypes of all six SNPs together (ie, rs35630000 — rs339I I 258 rs36046834 - rs33990840 - rs28529127-rs33933482, with major haplotype CTGGGG, $f=0.82$ ). The SNPs selected for analysis tagged all the other known, common SNP variation in the AVPRIB genes with $r^{2} \geqslant 0.80$ as follows: rs35630000 tagged rs34786964, rs28535253, rs35076969. —rs339I I 258 tagged rs33985287, rs287507।3, rs285।7619, rs2873398।, rs35394684, rs288707।7, rs28878533, rs28520962, rs61434100, rs28373064, rs33940624, rs28607590, rs35997092. —rs33990840 tagged rs28536160, rs33932943, rs35608965. rs28529I 27 tagged rs28632197, rs34327। 64, rs3397। I I9, rs35369693, rs33935503, rs28588803, rs28766023, rs358I0727, rs34792278. —rs33933482 tagged rs28499431, rs28425623, rs28380027, rs3883899, rs28483632, rs28477649, rs28676508, rs28452187, rs28575468, rs636। 1260.

${ }^{\mathrm{a}}$ On chromosome I, genome build 37.3/assembly GRCh37.

Rating Scale (Beck et al, 1975), the main outcome measure of the study. SA offspring were also assessed for depression and anxiety disorders and depressive symptoms. Lifetime diagnoses of depression and anxiety disorders were assessed with the Composite International Diagnostic Interview v2.1(World Health Organization 1997), according to ICD10 codes F32-F33, F34.1 (depression or dysthymia, $n=142$ SA offsprings) or F40-45 (neurotic, stress-related and somatoform disorders, $n=176 \mathrm{SA}$ offsprings). Self-report of current depressive symptoms with the Beck Depression Inventory (BDI; Beck et al, 1961) was completed at their homes by the subjects with the trained interviewer present in the same room, while taking precautions not to influence the subject replies, occurring after the diagnostic assessment and before the blood sampling. A BDI score $\geqslant 17$ was used to analyze subjects with 'high' current depressive symptoms ('high-BDI'; $n=298$ SA offsprings, $n=39$ HVs).Subjects were not under influence of alcohol or drugs during interview, and past year alcohol-drug use disorders were equally prevalent among low- and high-BDI subjects ( $\sim 20 \%)$. Last month (ie, predating the SA, 'pre-SA') use of (any) psychotropic medication was more prevalent in high(34\%) vs low-BDI subjects $(17 \% ; P=0.001)$. Furthermore, last month pre-SA use of selective serotonin re-uptake inhibitors (SSRIs) was more prevalent in high- (13\%) vs low-BDI subjects $(4 \% ; P<0.001)$. The same interview procedure was used for the HVs, ascertained for not having any history of lifetime SA or psychiatric diagnosis, after recruitment from workplaces in Odessa in 2003 and 2005. Secondary outcome measures used to characterize any 
findings are also described (Supplement). Complete BDI score questionnaires were available for $98.6 \%(n=651)$ of SA subjects.

\section{Statistical Analyses}

Analyses of Hardy-Weinberg equilibrium, genetic association, $\mathrm{G} \times \mathrm{E}$, effect size estimation, covariation with secondary outcomes, and power analysis has been described previously(Ben-Efraim et al, 2011; 2012; Sokolowski et al, 2012), with additional details in the Supplementary Methods. Briefly, analysis of additive, allelic genetic association and linkage was performed by using an additive model family-based association test in software FBAT v2.0.3 (http://www.biostat.harvard.edu/ fbat/default.html) and transmission disequilibrium test in Haploview v4.2(Barrett et al, 2005). Effect size estimates and genotype relative risks were assessed by the help of software package GENASSOC (Cordell and Clayton 2002) as implemented in STATA v11 (StataCorp, College Station, TX, USA), with likelihood-ratio test $P$-values reported for interactions. $\mathrm{G} \times \mathrm{Es}$ were also tested by using software fbat-i (Hoffmann et al, 2009). Haplotype association tests (Horvathet al, 2004) were performed on the haplotypes observed with the six selected SNPs, using a cutoff frequency $\geqslant 0.05$. Analysis of a lowfrequency SNP genetic effect was performed by aggregating heterozygotes and minor allele homozygotes and using Fisher's exact test (Asimit and Zeggini 2010). Tests of additive $\times$ additive $\mathrm{G} \times \mathrm{G}$ were performed using 15 pseudosibs per case (Cordell et al, 2004), as implemented in software trio (Schwender et al, 2011).

$P$-values (two-tailed) not corrected for multiple comparisons (nominal) are shown. The multiple tests performed in the analysis of the first hypothesis (i) $(n=54$ SNPs and $n=45$ haplotype tests) were corrected for by determining the number of independent variables by spectral decomposition (http://gump.qimr.edu.au/general/daleN/) (Nyholt 2004), revealing that 4.023 genetic variables had been investigated in three sample strata, yielding a corrected significance threshold of $P=0.00414$. After testing the second hypothesis (ii) of $\mathrm{G} \times \mathrm{Es}(n=129$ tests), all $P$-values $(n=228)$ generated in the study (Supplementary Figure S1) for hypothesis (i) + (ii) were included in false discovery rate (FDR) analysis (Strimmer 2008). Results that passed the first correction and that according to the false discovery rate did not contain any false positives, are herein referred to as significant main results. Odds ratios (ORs) with $80 \%$ power (using $\alpha=0.01$, log-additive model, $n=660$ trios, population risk $=0.01$ ) for the common SNPs analyzed here (Table 1) were in the range between 1.4-1.6 for the total sample and in the range between 1.6-1.9 for the sexstratified analyses $(0.18>\mathrm{MAF}>0.07)$. Further, power calculations also are shown in Supplementary Table S1.

\section{RESULTS}

$A V P R 1 B$ Genetic Variants show Direct association and Linkage on SAwith High Depressive Symptoms and with No role for $\mathrm{G} \times \mathrm{Es}$

To test the first hypothesis, we analyzed the family-based association and linkage of six selected SNPs, as well as 6-
SNP haplotypes thereof (Figure 1 and Table 1), on the outcomes of SA, SA with high current depressive symptoms (high-BDI), or SA with depression and anxiety disorders, among male and female separately or combined $(n=99$ tests). Table 2 shows the top associations observed after this analysis (at nominal $P<0.01$ ), wherein the top two $P$-values withstood correction for multiple testing by the number of statistically independent variables analyzed $(n=12.07)$. All findings with high-BDI described in Table 2 were also nominally significant $(P<0.05)$ in the total SA sample $(n=660$ trios; data not shown).To test the second hypothesis, we used the same genetic markers and SA outcomes as in the first hypothesis but instead analyzed the $\mathrm{G} \times$ Es using six different environmental exposures; high lifetime SLEs, high network-related SLEs, childhood/adolescence physical assault, childhood/adolescence sexual assault, combined childhood/adolescence assaults and high lifetime SLEs, as well as repeated childhood/adolescence or adulthood assaults, respectively ( $n=129$ tests). None of the $\mathrm{G} \times \mathrm{E}$ analyses revealed $P<0.01$, despite the reasonable power to observe such effects for many of the analyses performed (see Supplementary Methods and Supplementary Table S1).Finally, false discovery rate analysis of all $P$-values generated in the two hypotheses tested $(n=228$, Supplementary Figure S2), showed that the top four associations in Table 2 had $Q<0.25$ (ie, $<1$ false positive among them). The results together suggested $A V P R 1 B$ to be a candidate gene of interest in SA etiology characterized by high-BDI, with main results driven by direct associations of SNP rs33990840 and the 6-SNP haplotype, and with no significant role for $\mathrm{G} \times \mathrm{Es}$.

\section{Follow-Up Characterization of the Main Results}

We then conducted several follow-up analyses, investigating various other factors that might be relevant in relation to the main results reported, by eg., re-analyzing subgroups in relation to those factors or re-analysis in the presence of interaction-terms. Table 2 shows the results of analysis in low-BDI subjects, with results being non-significant, in contrast to the main results for high-BDI. This relative difference was the greatest for the main result with SNP rs33900840 (Table 2; $P_{\mathrm{LRT}}<0.05$ ). We further observed that the main results with high-BDI were driven by the borderline-to-moderate ranges of clinically interpreted depressive symptoms (Figure 2).

The main results were best fit by the additive model (data not shown), as was used in the hypothesis testing stage. The allelic direction and additive effect of SNP rs33990840 was also confirmed in a less-powered case-control re-analysis for high-BDI SA outcome (OR = 1.74). More detailed sliding window haplotype analysis showed multiple 2-SNP haplotypes with similar frequency, allelic direction, and significance, being representative of the main results with SNP rs33990840 (data not shown). Two 2-SNP haplotypes of SNPs rs33990840-rs33911258, showed particularly parsimonious minor $v s$ major allele and under- $v s$ overtransmissions, respectively, comparable to those observed with the SNPs (Tables 2 vs 3).

Sex effects did not significantly contribute to the main results (data not shown). Although, eg, results for SNPs rs33900840 and rs33911258 were nominally significant in 
Table 2 AVPRIB Family-Based Associations Observed on High Depressive Symptoms in SA, by Multiple Genetic Markers

\begin{tabular}{|c|c|c|c|c|c|c|c|c|c|}
\hline \multirow[t]{2}{*}{$\begin{array}{l}\text { Genetic } \\
\text { marker }\end{array}$} & \multirow[t]{2}{*}{ Sex } & \multirow[t]{2}{*}{ Outcome } & \multicolumn{4}{|c|}{ Results for outcome } & \multicolumn{3}{|c|}{$\begin{array}{c}\text { Results for } \\
\text { non-outcome }\end{array}$} \\
\hline & & & $N$ & $\begin{array}{l}\text { Major: minor } \\
\text { transmitted } \\
\text { alleles } \\
(P \text {-value })\end{array}$ & $\begin{array}{l}\text { Over- } \\
\text { transmitted } \\
\text { allele }\end{array}$ & $\begin{array}{c}\text { OR } \\
(95 \% \mathrm{Cl})\end{array}$ & $N$ & $\begin{array}{c}\text { Major: minor } \\
\text { transmitted } \\
\text { alleles } \\
(P \text {-value })\end{array}$ & OR $(95 \% \mathrm{Cl})$ \\
\hline rs339|1258 & $m+f$ & $\mathrm{SA}$ and high-BDI & 294 & I I 0 T: 72 C (0.0049) & Major ( $\mathrm{T}$ ) & $1.53(1.14 ; 2.06)$ & 357 & $109 \mathrm{T:} 94 \mathrm{C}(0.29)$ & $1.16(0.88 ; 1.53)$ \\
\hline rs339|1258 & $m+f$ & All SA & 660 & $\begin{array}{c}220 \mathrm{~T}: \text { I I } 68 \mathrm{C} \\
(0.0083)\end{array}$ & Major (T) & $1.31(1.07 ; 1.60)$ & NA & NA & NA \\
\hline rs36046834 & $\mathrm{m}$ & $\mathrm{SA}$ and high-BDI & 137 & 9 G: I C (0.0084) & Major (G) & $9.00(1.14 ; 71.0)$ & 193 & 4 G :7 C (0.37) & $0.57(0.17 ; 1.92)$ \\
\hline
\end{tabular}

Abbreviations: $\mathrm{m}$, analysis in male offspring; $\mathrm{m}+\mathrm{f}$, analysis in male and female offspring; $\mathrm{N}$, number of trios; $\mathrm{NA}$, not available; OR, odds ratio; $\mathrm{SA}$ and high-BDI, subgroup of suicide attempt (SA) with a high Beck Depression Inventory score of $\geqslant 17$.

Notes: All family-based associations with a nominal $P<0.01$ are shown, as was observed after testing the first hypothesis.

aRefers to follow-up characterization in SA offspring with a low Beck Depression Inventory score of $<17$.

${ }^{b}$ Below the Bonferroni-corrected significance threshold of $P=0.0041$.

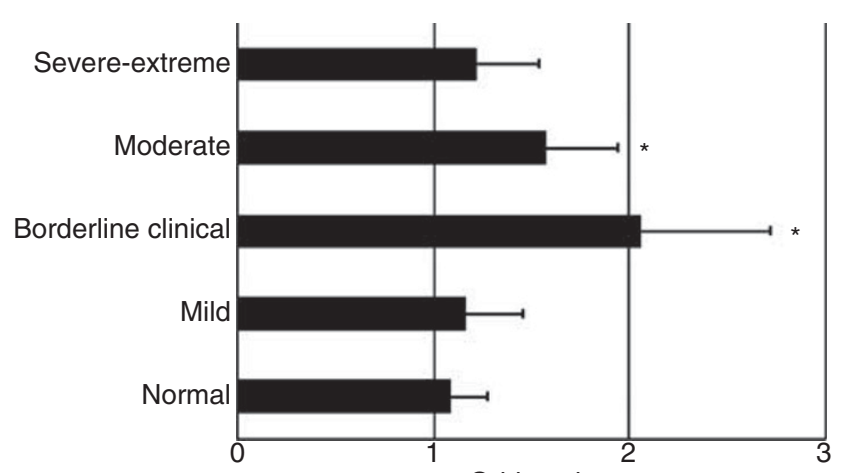

Odds ratio

(allele transmission)

Figure 2 The main genetic associations identified were in the range between borderline clinical and moderate depressive symptoms in suicide attempt (SA). Follow-up characterization of the 6-SNP haplotype is shown along more detailed, clinically interpreted cutoffs on the Beck Depression Inventory (BDI) score scale, with results being representative also for single nucleotide polymorphisms (SNPs) rs33990840 and rs339II258 (not shown here). The family-based association analysis was conducted using trios, with bars showing the allelic transmission odds ratios ( \pm SE) for SA offspring in either the normal (BDI score $0-10 ; n=248$ ), mild (BDI score ||$-\mid 6 ; n=109)$, borderline clinical (BDI score 17-20; $n=7 \mid$ ), moderate (BDI score $21-30 ; n=128)$, or severe-extreme (BDI score $21-30 ; n=95)$ range of current depressive symptoms. $* P<0.05$.

males for high-BDI in SA, they were observed with significance in the males and females combined (Table 2). The minor result for low-frequency SNP rs36046834 was observed only in male high-BDI offspring (Table 2) but on the background that SNPs rs36046834 and rs33990840 were equivalent in 29 out of 30 individuals (data not shown). For the last month pre-SA use of any kind of psychotropic medications or SSRIs, past year alcohol-drug use disorders, or the presence of $12.9 \%$ psychotic subjects in the sample, the main results were retained in absence of those factors $(P<0.05)$ and with no covariate interaction observed $\left(P_{\text {LRT }}>0.05\right)$. Nevertheless, the ORs for SNPs rs33990840 and rs33911258 on high-BDI were elevated (compared with
Table 2) among subjects with last month pre-SA use of any psychotropic medications (ORs 2.6 and 2.1, respectively) or SSRIs (ORs 3.3 and 2.5, respectively). Concerning further SA-related secondary outcome measures (Supplement), the SA subjects driving the main result of SNP rs33990840 (Table 2) could also be characterized as having used a nonviolent SA method $\left(P_{\mathrm{LRT}}=0.0047\right)$ and by scoring below the sample median on the Trait Anger Scale $\left(P_{\mathrm{LRT}}=0.03\right)$.

\section{Exploratory $\mathrm{G} \times \mathrm{G}$ Analyses Between $A V P R 1 B$ and CRHR1}

Screening through $\mathrm{G} \times \mathrm{Gs}$ on all $\mathrm{SA}$ outcomes investigated herein failed to indicate any putative interaction effects between $A V P R 1 B$ and CRHR1 SNPs (all P>0.05; data not shown), despite the reasonable power to observe such effects for many of the analyses conducted (see Supplementary Methods and Supplementary Table S1)

\section{DISCUSSION}

This study showed for the first time that $A V P R 1 B$ variants were linked and associated with SA characterized by highBDI, mainly driven by association of the previously unreported exon 1 SNP rs33990840 and a 6-SNP haplotype representing all known common SNP variation in AVPR1B. The haplotypes over- and under-transmitted reflected the transmission distortion observed with the all single-SNP analyses (Tables 2 and 3 and text), whereby major alleles could be interpreted as 'risk' and minor alleles as 'protective' alleles, respectively. According to LD analysis and haplotype mapping, those risk $v s$ protective allelic effects on high-BDI SA were captured most parsimoniously by using corresponding 2-SNP haplotypes of SNPs rs33911258 and rs33990840 (Table 3).

Other potentially interesting SNPs were tagged by our associated SNPs (Table 1), according to a novel EUR reference panel for $A V P R 1 B$ (The 1000 Genomes Project 
Table 3 Haplotypes Reflecting the SNP Associations on SA and Depressive Symptoms in SA

\begin{tabular}{lcccc}
\hline $\begin{array}{l}\text { Haplotype } \\
\text { rs33911258: } \\
\text { rs33990840 }\end{array}$ & Outcome Frequency & $\begin{array}{c}\text { Informative } \\
\text { transmissions } \\
\text { column I: other } \\
\text { haplotypes }\end{array}$ & $\begin{array}{c}\text { HBAT } \\
\text { P-value }\end{array}$ \\
\hline $\begin{array}{l}\text { Major T-allele:major } \\
\text { G-allele }\end{array}$ & SA & 0.823 & $220: 168$ & 0.0067 \\
& BDI & 0.823 & $110: 72$ & 0.0034 \\
$\begin{array}{l}\text { Minor C-allele:major } \\
\text { G-allele }\end{array}$ & SA & 0.103 & $110: 133$ & 0.1401 \\
& BDI & 0.103 & $49: 60$ & 0.2921 \\
$\begin{array}{l}\text { Minor C-allele:minor } \\
\text { C-allele }\end{array}$ & SA & 0.074 & $76: 105$ & 0.0248 \\
& BDI & 0.074 & $29: 56$ & 0.0021 \\
\hline
\end{tabular}

Abbreviations: BDI, SA with Beck Depression Inventory score $\geqslant 17$;

HBAT, family-based haplotype association test; SA, suicide attempt.

Calculations in the total sample.

Consortium 2010). Minor alleles of rs33985287 and rs28536160 were protective in females in a previous association and linkage study on childhood-onset mood disorders (Dempster et al, 2007) and rs28536160 minor allele was protective on bipolar disorder with psychotic features (Leszczynska-Rodziewicz et al, 2012). These results were in the same allelic direction as we observed with SNPs rs33911258 and rs33990840, which tag rs33985287 and rs28536160, respectively (Table 1). SNP rs28536160 was also genotyped herein (Table 1) and a transmission distortion in the same allelic direction was confirmed (data not shown). A risk effect of the major allele haplotype in trios was also congruent with Dempster et al (2007). In contrast to Dempster et al (2007), however, the associations were observed here without sex specificity. The results herein were not congruent with other previously observed SNP associations on recurrent major depression (van West $e t$ al, 2004), panic disorder (Keck et al, 2008), and attentiondeficit hyperactivity disorder (van West et al, 2009), and a protective effect of a major allele haplotype in two of these studies (van West et al, 2004; van West et al, 2009).

Our results most closely match those of Dempster et al (2007). Perhaps this may be explained by similar methods and outcomes: both Dempster et al (2007) and this study used family-based designs and therefore the associations were robust to population stratification, and childhoodonset major depression is an established endophenotype of suicidal behavior (Mann et al, 2009). Ethnic differences between studies may also be important in the absence of population stratification. Differential ethnicity-dependent selection signatures on $A V P R 1 B$ were reported, suggesting that $A V P R 1 B$ was subjected to natural selection during human evolutionary history and balancing selection in Europeans (Cagliani et al, 2009). A study in an ethnically diverse sample did not observe an association with antidepressant treatment outcome (Binder et al, 2010). Major allele risk effects congruent to our results were observed across psychiatric outcomes in Hungarians (Dempster et al, 2007), Poles (Leszczynska-Rodziewicz et al, 2012), and Ukrainians (here) vs major allele protective effects in Belgians (van West et al, 2004; van West et al, 2009) and Swedes (van West et al, 2004) and a recessive risk effect in Germans (Keck et al, 2008). This may be supported by intra-European patrilineal haplogroup clines that are separated by central European geographic boundaries (Rosser et al, 2000). Notably, genetic boundaries were determined to a greater extent by geographic rather than linguistic boundaries, ie, genetic boundaries between Hungary and its neighbors were not significant relative to continental geographic boundaries (Rosser et al, 2000). In separate studies, Poles and Ukrainians were found to share a common patrilineal heritage together with southwestern Russians (Balanovsky et al, 2008) while Poles and Germans were genetically separated according to the present political boundary (Kayser et al, 2005). Nevertheless, intraEuropean selection signatures on AVPR1B have not been studied and thus any correlation with patrilineal boundaries is speculative. Also, not all results here were congruent with Dempster et al (2007), ie, we did not observe significance in the total sampleof non-synonymous exon 2 SNP rs35369693 (tagged by rs28529127 and NS for an allelic test in the total sample and by sex in this study). However, rs35369693 was imputed here with relatively lower accuracy (Figure 1) while sex-specific genotypic effects were not tested due to insufficient transmissions.

\section{Potential Causal Roles of Associated SNPs in Pathogenesis of Depressive Symptoms in SA}

This study showed novel direct association of rs33990840 on SA and high current depressive symptoms in SA. Although the study design did not permit us to exclude other unknown environmental influences during the interviews that might have influenced the BDI score assessments, we found no reasons as to why such influences would occur non-randomly (causing bias) and the dichotomization of BDI scores should insulate against such influences on particular BDI items. However, the last month pre-SA use of SSRIs may have lowered the range of BDI scores we observed among the SA subjects who were driving the association in high-BDI (Figure 2 and text). Rs33990840 translates to $191 \mathrm{Gly}>\mathrm{Arg}$, which was previously predicted to affect receptor activation according to its position in the second extracellular loop (Supplementary Figure S1) and proximity to two putative AVP-binding residues (Cagliani et al, 2009). This study supports the functional prediction by showing rs33990840 transmission distortion and altered risk for having depressive symptoms in SA.

The associated SNPs may instead be in high LD with functional variants. Both rs33990840 and rs33911258 are in extensive LD with SNPs in the $5^{\prime}$ flanking region (Figure 1 and Table 1), which contains several sequence motifs that are highly conserved between human, rat, and mouse, suggesting a role in $A V P R 1 B$ expression (Rene and de Keyzer 2002). Specifically in humans, the $5^{\prime}$ region contains an open reading frame, putative transcription factor binding sites for sex and stress hormones, and sequence for a spliced antisense transcript that is expressed in pituitary and which may affect $A V P R 1 B$ transcription and/ or translation (Sugimoto et al, 1994; Rene et al, 2000).

\section{AVPR1B $\mathrm{G} \times \mathrm{E} v s$ Genetic Associations}

A previous study found no association of AVPR1B SNPs on DEX suppression in response to an acute psychosocial 
stressor (van West et al, 2010). Putative $A V P R 1 B \times$ SLEs or $A V P R 1 B \times C R H R 1$ were not observed here, despite several of these tests achieving sufficient power (Supplementary Table S3), as shown also by our previous investigations, eg, of CRHR1 with multiple significant $\mathrm{G} \times$ Es observed (BenEfraim et al, 2011).Taken together, this suggests that $A V P R 1 B$ common SNPs did not moderate the effect of SLEs either directly or indirectly via SLE-moderating CRHR 1 variants and that the genetics of $A V P R 1 B$ vs CRHR 1 on SA in our sample may be distinct. As inferred by having observed no SLE-moderating effects, a role for $A V P R 1 B$ common SNPs in stress reactivity was not supported here.

However, we did observe direct genetic associations with SA. Accumulated observations in our sample and others shows that HPA axis regulatory genes, as well as other genes, are directly associated with suicidal behavior (Wasserman et al, 2007; Brezo et al, 2008), in line with a diversified genetic etiology. It is noteworthy that we have previously observed genetic associations (in the currently used trio data set) of common SNPs in neural conduction and neurotransmitter release (Wasserman et al, 2005), monoaminergic signaling (Wasserman et al, 2007; BenEfraim et al, 2012), and glutamatergic signaling genes (Sokolowski et al, 2012). Interestingly, potential functional roles that are not directly related to mediating stress reactivity in depression and suicidal behavior have been shown for dysregulation of AVP (Merali et al, 2006; Mlynarik et al, 2007; Bao and Swaab 2010), but the role of $A V P R 1 B$ genetics is still poorly understood.

\section{CONCLUSION}

$A V P R 1 B$ variants were associated with SA for the first time, predominantly concurrent with high depressive symptoms. The observed associations are congruent with a previous family-based study on childhood-onset mood disorders (Dempster et al, 2007), although sex specificity was not observed here. No role for common AVPR1B SNPs, as effect-modifiers of certain SLEs or CRHR1 variants in $\mathrm{SA}$, or depression and anxiety in SA was observed. Future studies of $A V P R 1 B$ genetic variation and stress-related outcomes would benefit from studies in other ethnic populations and testing sex-specificity. Equally interesting are the polymorphisms with regard to the HPA axis function and potentially AVPR1B-CRHR1 heterodimerization. Finally, it would be interesting to know whether 191 Gly $>$ Arg (rs 33990840) changes the pharmacological (antidepressant) response to AVPR1B antagonists.

\section{ACKNOWLEDGEMENTS}

We thank all interviewers at the Human Ecological Health Organization/Odessa National Mechnikov University, Odessa, Ukraine; Professor Vsevolod Rozanov for coordination of the material collection in Ukraine; Dr Vladymyr Bogatov and laboratory technician Lars Holmberg for technical assistance; Dr Tatyana Reytarova for logistical assistance; all those who have given their consent to participate as research subjects in this study; and the SNP\&SEQ facility in Uppsala, Sweden. This study was funded by the Knut and Alice Wallenberg Foundation, the
Marianne and Marcus Wallenberg Foundation, and the American Foundation for Suicide Prevention.

\section{DISCLOSURE}

The authors declare no conflict of interests.

\section{REFERENCES}

Asimit J, Zeggini E (2010). Rare variant association analysis methods for complex traits. Annu Rev Genet 44: 293-308.

Balanovsky O, Rootsi S, Pshenichnov A, Kivisild T, Churnosov M, Evseeva I et al (2008). Two sources of the Russian patrilineal heritage in their Eurasian context. Am J Human Genet 82: 236-250.

Bao AM, Swaab DF (2010). Corticotropin-releasing hormone and arginine vasopressin in depression focus on the human postmortem hypothalamus. Vitam Horm 82: 339-365.

Bardeleben U, Holsboer F (1989). Cortisol response to a combined dexamethasone-human corticotrophin-releasing hormone challenge in patients with depression. J Neuroendocrinol 1: 485-488.

Barrett JC, Fry B, Maller J, Daly MJ (2005). Haploview: analysis and visualization of $\mathrm{LD}$ and haplotype maps. Bioinformatics 21: 263-265.

Beck AT, Beck R, Kovacs M (1975). Classification of suicidal behaviors: I. Quantifying intent and medical lethality. $A m \mathrm{~J}$ Psychiatry 132: 285-287.

Beck AT, Ward CH, Mendelson M, Mock J, Erbaugh J (1961). An inventory for measuring depression. Arch Gen Psychiatry 4: 561-571.

Ben-Efraim YJ, Wasserman D, Wasserman J, Sokolowski M (2011). Gene-environment interactions between CRHR1 variants and physical assault in suicide attempts. Genes Brain Behav 10: 663-672.

Ben-Efraim YJ, Wasserman D, Wasserman J, Sokolowski M (2012). Family-based study of HTR2A in suicide attempts: observed gene, gene $\mathrm{x}$ environment and parent-of-origin associations. $\mathrm{Mol}$ Psychiatry (doi:10.1038/mp.2012.86).

Binder EB, Nemeroff CB (2010). The CRF system, stress, depression and anxiety-insights from human genetic studies. Mol Psychiatry 15: 574-588.

Binder EB, Owens MJ, Liu W, Deveau TC, Rush AJ, Trivedi MH et al (2010). Association of polymorphisms in genes regulating the corticotropin-releasing factor system with antidepressant treatment response. Arch Gen Psychiatry 67: 369-379.

Brezo J, Klempan T, Turecki G (2008). The genetics of suicide: a critical review of molecular studies. Psychiatr Clin North Am 31: 179-203.

Browning BL, Browning SR (2009). A unified approach to genotype imputation and haplotype-phase inference for large data sets of trios and unrelated individuals. Am J Human Genet 84: 210-223.

Cagliani R, Fumagalli M, Pozzoli U, Riva S, Cereda M, Comi GP et al (2009). A complex selection signature at the human AVPR1B gene. BMC Evol Biol 9: 123.

Cordell HJ, Barratt BJ, Clayton DG (2004). Case/pseudocontrol analysis in genetic association studies: a unified framework for detection of genotype and haplotype associations, gene-gene and gene-environment interactions, and parent-of-origin effects. Genet Epidemiol 26: 167-185.

Cordell HJ, Clayton DG (2002). A unified stepwise regression procedure for evaluating the relative effects of polymorphisms within a gene using case/control or family data: application to HLA in type 1 diabetes. Am J Human Genet 70: 124-141.

Dempster EL, Burcescu I, Wigg K, Kiss E, Baji I, Gadoros J et al (2007). Evidence of an association between the vasopressin V1b receptor gene (AVPR1B) and childhood-onset mood disorders. Arch Gen Psychiatry 64: 1189-1195. 
Griebel G, Stemmelin J, Gal CS, Soubrie P (2005). Non-peptide vasopressin V1b receptor antagonists as potential drugs for the treatment of stress-related disorders. Curr Pharm Des 11: $1549-1559$.

Hoffmann TJ, Lange C, Vansteelandt S, Laird NM (2009). Geneenvironment interaction tests for dichotomous traits in trios and sibships. Genet Epidemiol 33: 691-699.

Horvath S, Xu X, Lake SL, Silverman EK, Weiss ST, Laird NM (2004). Family-based tests for associating haplotypes with general phenotype data: application to asthma genetics. Genet Epidemiol 26: 61-69.

Kayser M, Lao O, Anslinger K, Augustin C, Bargel G, Edelmann J et al (2005). Significant genetic differentiation between Poland and Germany follows present-day political borders, as revealed by Y-chromosome analysis. Hum Genet 117: 428-443.

Keck ME, Kern N, Erhardt A, Unschuld PG, Ising M, Salyakina D et al (2008). Combined effects of exonic polymorphisms in CRHR1 and AVPR1B genes in a case/control study for panic disorder. Am J Med Genet B Neuropsychiatr Genet 147B: 1196-1204.

Leszczynska-Rodziewicz A, Szczepankiewicz A, Dmitrzak-Weglarz M, Skibinska M, Hauser J (2012). Association between functional polymorphism of the AVPR1b gene and polymorphism rs1293651 of the CRHR1 gene and bipolar disorder with psychotic features. J Affect Disord 138: 490-493.

Lönnqvist J (2009). Major psychiatric disorders in suicide and suicide attempters. In: Wasserman DWasserman C (eds) Oxford Textbook of Suicidology and Suicide Prevention: A Global Perspective. Oxford University Press Inc: New York, NY, USA, pp 275-286.

Mann JJ, Arango VA, Avenevoli S, Brent DA, Champagne FA, Clayton P et al (2009). Candidate endophenotypes for genetic studies of suicidal behavior. Biol Psychiatry 65: 556-563.

Mann JJ, Currier D, Stanley B, Oquendo MA, Amsel LV, Ellis SP (2006). Can biological tests assist prediction of suicide in mood disorders? Int J Neuropsychopharmacol 9: 465-474.

Merali Z, Kent P, Du L, Hrdina P, Palkovits M, Faludi G et al (2006). Corticotropin-releasing hormone, arginine vasopressin, gastrin-releasing peptide, and neuromedin $\mathrm{B}$ alterations in stress-relevant brain regions of suicides and control subjects. Biol Psychiatry 59: 594-602.

Mlynarik M, Zelena D, Bagdy G, Makara GB, Jezova D (2007). Signs of attenuated depression-like behavior in vasopressin deficient Brattleboro rats. Horm Behav 51: 395-405.

Murat B, Devost D, Andres M, Mion J, Boulay V, Corbani M et al (2012). V1b and CRHR1 receptor heterodimerization mediates synergistic biological actions of vasopressin and CRH. Mol Endocrinol 26: 502-520.

Nyholt DR (2004). A simple correction for multiple testing for single-nucleotide polymorphisms in linkage disequilibrium with each other. Am J Hum Genet 74: 765-769.

Purba JS, Hoogendijk WJ, Hofman MA, Swaab DF (1996). Increased number of vasopressin- and oxytocin-expressing neurons in the paraventricular nucleus of the hypothalamus in depression. Arch Gen Psychiatry 53: 137-143.

Rene P, de Keyzer Y (2002). The vasopressin receptor of corticotroph pituitary cells. Prog Brain Res 139: 345-357.

Rene P, Lenne F, Ventura MA, Bertagna X, de Keyzer Y (2000). Nucleotide sequence and structural organization of the human vasopressin pituitary receptor (V3) gene. Gene 241: 57-64.
Roper J, O'Carroll AM, Young W 3rd, Lolait S (2011). The vasopressin Avpr1b receptor: molecular and pharmacological studies. Stress 14: 98-115.

Rosser ZH, Zerjal T, Hurles ME, Adojaan M, Alavantic D, Amorim A et al (2000). Y-chromosomal diversity in Europe is clinal and influenced primarily by geography, rather than by language. $\mathrm{Am}$ J Hum Genet 67: 1526-1543.

Schwender H, Taub MA, Beaty TH, Marazita ML, Ruczinski I (2012). Rapid testing of SNPs and gene-environment interactions in case-parent trio data based on exact analytic parameter estimation. Biometrics 68: 766-773.

Scott LV, Dinan TG (1998). Vasopressin and the regulation of hypothalamic-pituitary-adrenal axis function: implications for the pathophysiology of depression. Life Sci 62: 1985-1998.

Sokolowski M, Ben-Efraim YJ, Wasserman J, Wasserman D (2012). Glutamatergic GRIN2B and polyaminergic ODC1 genes in suicide attempts: associations and gene-environment interactions with childhood/adolescent physical assault. Mol Psychiatry (doi:10.1038/mp.2012.112).

Strimmer K (2008). fdrtool: a versatile R package for estimating local and tail area-based false discovery rates. Bioinformatics 24: $1461-1462$

Sugimoto T, Saito M, Mochizuki S, Watanabe Y, Hashimoto S, Kawashima H (1994). Molecular cloning and functional expression of a cDNA encoding the human V1b vasopressin receptor. $J$ Biol Chem 269: 27088-27092.

The 1000 Genomes Project Consortium (2010). A map of human genome variation from population-scale sequencing. Nature 467: 1061-1073.

van West D, Del-Favero J, Aulchenko Y, Oswald P, Souery D, Forsgren T et al (2004). A major SNP haplotype of the arginine vasopressin 1B receptor protects against recurrent major depression. Mol Psychiatry 9: 287-292.

van West D, Del-Favero J, Deboutte D, Van Broeckhoven C, Claes S (2009). Arginine vasopressin receptor gene-based single-nucleotide polymorphism analysis in attention deficit hyperactivity disorder. Psychiatr Genet 19: 102-103.

van West D, Del-Favero J, Deboutte D, Van Broeckhoven C, Claes S (2010). Associations between common arginine vasopressin $1 \mathrm{~b}$ receptor and glucocorticoid receptor gene variants and HPA axis responses to psychosocial stress in a child psychiatric population. Psychiatry Res 179: 64-68.

Wasserman D, Geijer T, Rozanov V, Wasserman J (2005). Suicide attempt and basic mechanisms in neural conduction: Relationships to the SCN8A and VAMP4 genes. Am J Med Genet B: Neuropsychiatr Genet 133B: 116-119.

Wasserman D, Geijer T, Sokolowski M, Rozanov V, Wasserman J (2007). Nature and nurture in suicidal behavior, the role of genetics: some novel findings concerning personality traits and neural conduction. Physiol Behav 92: 245-249.

Wasserman D, Sokolowski M, Rozanov V, Wasserman J (2008). The CRHR1 gene: a marker for suicidality in depressed males exposed to low stress. Genes Brain Behav 7: 14-19.

World Health Organization (1997). Composite International Diagnostic Interview (CIDI, version 2.1).. WHO: Geneva, Switzerland.

Young SF, Griffante C, Aguilera G (2007). Dimerization between vasopressin $\mathrm{V} 1 \mathrm{~b}$ and corticotropin releasing hormone type 1 receptors. Cell Mol Neurobiol 27: 439-461.

Supplementary Information accompanies the paper on the Neuropsychopharmacology website (http://www.nature.com/npp) 